\title{
Neue Schriften
}

Bibliographie zur Ur- und Frühgeschichte

1. 3. 1987 bis 29.2 .1988 mit Nachträgen

Einführung . 277

\section{Entwicklung und Methode der Forschung}

1. Methodik der Archäologie . . . . . . . . . . . . . . . . . . . 277

2. Grabungstechnik, Fundbergung, Präparation, Konservierung, Restauration 278

3. Museumswesen und Bodendenkmalpflege . . . . . . . . . . . . . . 279

4. Geschichte der Archäologie, Biographien und Nekrologe . . . . . . . . . 279

5. Neue Zeitschriften, Fest- und Gelegenheitsschriften, Sammelwerke . . . . 280

6. Bibliographien . . . . . . . . . . . . . . . . . . . . . . . . 280

7. Instituts- und Tagungsberichte . . . . . . . . . . . . . . . . . . . . . . 281

\section{Ergebnisse der archäologischen Forschung}

A. Allgemeine Arbeiten . . . . . . . . . . . . . . . . . . . . . . . . . . 282

B. Zeitliche Ubersichten . . . . . . . . . . . . . . . . . . . . . . . . 283

1. Paläolithikum . . . . . . . . . . . . . . . . . . . . . . . . 283

2. Mesolithikum . . . . . . . . . . . . . . . . . . . . . . . . 284

3. Neolithikum . . . . . . . . . . . . . . . . . . . . . . . . . 285

4. Bronzezeit . . . . . . . . . . . . . . . . . . . . . . . . . . 286

5. Vorrömische Eisenzeit . . . . . . . . . . . . . . . . . . . . . 287

6. Römische Kaiserzeit . . . . . . . . . . . . . . . . . . . . . . . . . . . . . 289

7. Völkerwanderungszeit . . . . . . . . . . . . . . . . . . . . . 290

8. Frühes Mittelalter . . . . . . . . . . . . . . . . . . . . . . . 291

C. Regionale Ubersichten

1. Berlin und Brandenburg (Bezirke Potsdam, Cottbus, Frankfurt/Oder). Zusammengestellt von F. Sărăteanu-Müller, Potsdam . . . . . . . . . . . . . . 293

2. Mecklenburg (Bezirke Rostock, Schwerin, Neubrandenburg). Zusammengestellt von S. Schacht, Schwerin . . . . . . . . . . . . . . . . . 295

3. Sachsen (Bezirke Dresden, Leipzig, Karl-Marx-Stadt). Zusammengestellt von H. Kaufmann, Dresden . . . . . . . . . . . . . . . . . . . 298

4. Sachsen-Anhalt (Bezirke Halle, Magdeburg). Zusammengestellt von $V$. Schneider, Halle . . . . . . . . . . . . . . . . . . . . . . . 301

5. Thüringen (Bezirke Erfurt, Gera, Suhl). Zusammengestellt von K. Peschel, Jena 


\section{Beiträge von Nachbarwissenschaften}

1. Physik und Chemie . . . . . . . . . . . . . . . . . . . . . . 305

2. Mineralogie und Metallurgie . . . . . . . . . . . . . . . . . . . . 306

3. Bodenkunde . . . . . . . . . . . . . . . . . . . . . . . . . 306

4. Quartärgeologie . . . . . . . . . . . . . . . . . . . . . . . 306

5. Botanik . . . . . . . . . . . . . . . . . . . . . . . . . . . 306

6. Zoologie. Zusammengestellt von H.-H. Müller, Berlin . . . . . . . . . . 307

7. Anthropologie. Zusammengestellt von H. Ullrich, Berlin . . . . . . . . . 309

8. Siedlungsgeographie und -geschichte. Zusammengestellt von E. Gringmuth-

Dallmer, Berlin . . . . . . . . . . . . . . . . . . . . . . . . 311

\section{Verschiedenes}

Grabungskalender 1988 . . . . . . . . . . . . . . . . . . . . . . . 106

Kleine Mitteilungen . . . . . . . . . . . . . . . . . . 49, $460,215,263,313$

Berichtigung: In Heft 4 (1988) muß es auf S. 168 in der 30 . Zeile von oben richtig heißen: „... mehr Frauen als Männer sterben ...“ 
Zeitschrift "Ausgrabungen und Funde“

Herausgeber: Zentralinstitut fur Alte Geschichte und Archäologie

der Akademie der Wissenschaften der Deutschen Demokratischen Republik.

Verlag: Akademie-Verlag Berlin, Leipziger Straße 3-4. Postfach 1233, DDR - 1086 Berlin;

Fernruf: 2236229 und 22362 01. Telex-Nr.: 114420; Bank: Staatsbank der DDR, Berlin, Kto.-Nr.: 6836-26-207 12.

Chefredakteur: Prof. Dr. phil. habil. Joachim Herrmann.

Anschrift der Redaktion: Zentralinstitut für Alte Geschichte und Archäologie der AdW, Leipziger Straße 3-4, Postfach 1310, DDR - 1086 Berlin, Fernruf 2236266.

Veröffentlicht unter der Lizenznummer 1337 des Presseamtes beim Vorsitzenden des Ministerrates der Deutschen Demokratischen Republik.

Gesamtherstellung: VEB Druckhaus „Maxim Gorki“, DDR - 7400 A1tenburg.

Erschelnungsweise: Dle Zeitschrift erscheint zweimonatlich. Die Jährlich erscheinenden 6 Hefte bilden einen Band. Bezugspreis je Band 60,- DM zuzüglich Versandspesen. Preis je Heft 10,-DM. Der gültige Jahresbezugspreis für die DDR ist der Postzeitungsliste zu entnehmen.

(c) 1988 by Akademie-Verlag Berlin . Printed in the German Democratic Republic.

AN (EDV) 16920 


\section{AUSGRABUNGEN}

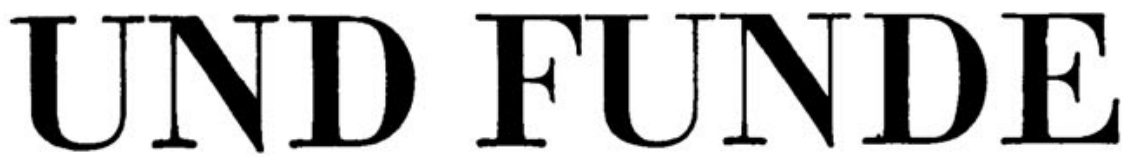

Archáologísche Beríchte und Informationen

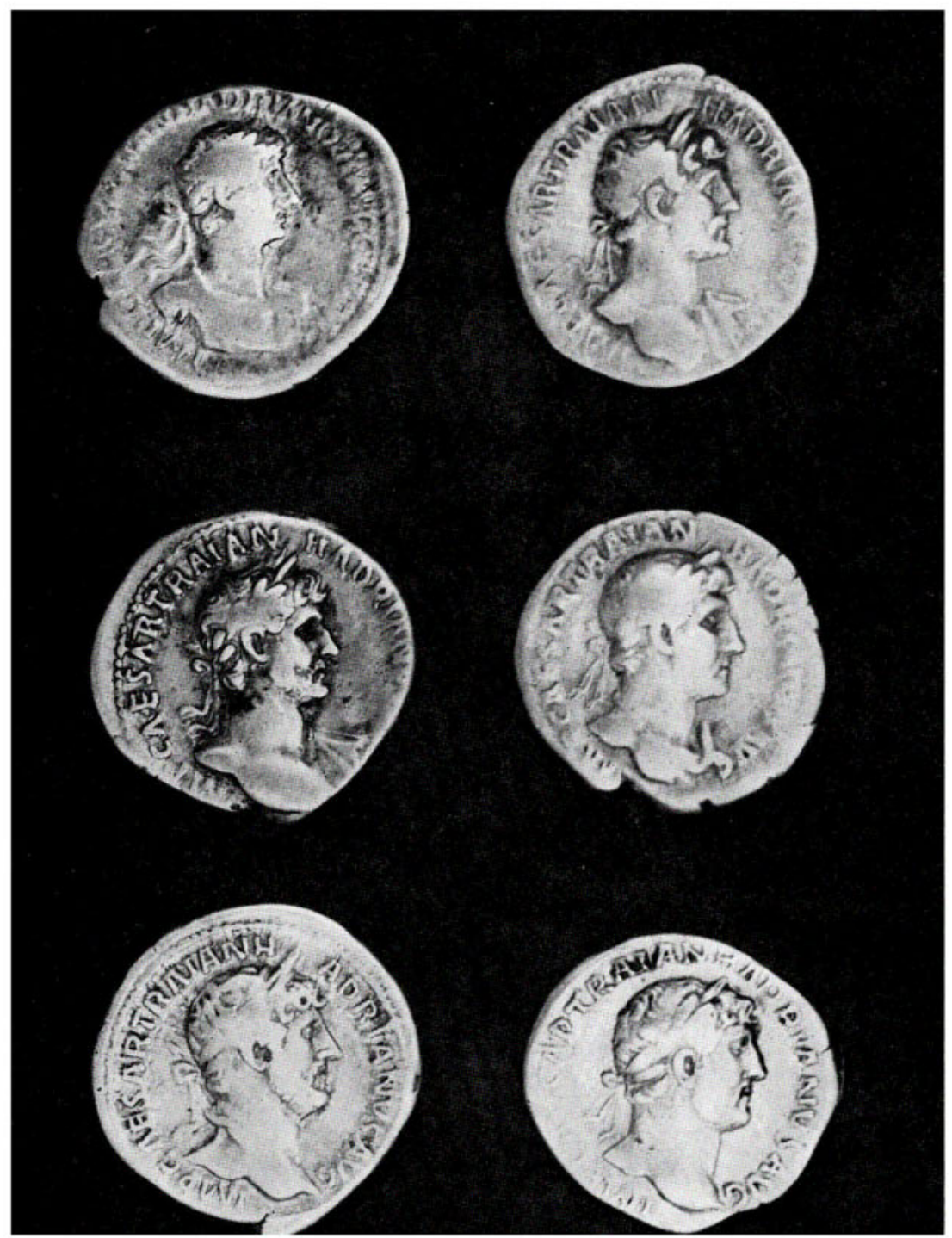

BAND 33

1988

HEFT 1

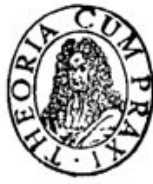

Akademie -

Derlag

Berlín

ISSN 0004-8127

Ausgrab. Funde

Berlin 33 (1988) 1, 1-52

EVP $3,-$ M 
Bezugsmöglichkeiten:

Bestellungen sind zu richten:

- in der $D D R$ an den Postzeitungsvertrieb unter Angabe der Kundennummer des Bestellenden oder an den Akademie-Verlag Berlin, Leipziger Straße 3-4, Postfach-Nr. 1233, DDR - 1086 Berlin;

- im sozialistischen Ausland an eine Buchhandlung für fremdsprachige Literatur oder an den zuständigen Postzeitungsvertrieb;

- in der BRD und Berlin (West) an eine Buchhandlung oder an die Auslieferungsstelle KUNST UND WISSEN, Erich Bieber OHG, Wilhelmstraße 4-6, D - 7000 Stuttgart 1;

- in den übrigen westeuropäischen Ländern an eine Buchhandlung oder an die Auslieferungsstelle KUNST UND WISSEN, Erich Bieber GmbH, General Wille-Str. 4, CH - 8002 Zürich;

- im übrigen Ausland an den Internationalen Buch- und Zeitschriftenhandel; den Buchexport, Volkseigener Außenhandelsbetrieb der Deutschen Demokratischen Republik, Postfach 160, DDR - 7010 Leipzig; oder an den Akademie-Verlag Berlin,

Leipziger Straße 3-4, Postfach-Nr. 1233, DDR - 1086 Berlin.

Verantwortlicher Redakteur: Joachim Herrmann

Redaktionssekretär: Hiltrud Heinrich

Redaktion: Peter Donat, Rudolf Feustel, Bernhard Gramsch, Heinz Grünert,

Dieter Kaufmann, Horst Keiling, Joachim Preuß, Heinz Seyer, Heinz-Joachim Vogt

Redaktionsbeirat: Günter Behm-Blancke, Werner Coblenz, Hans Grimm, Paul Grimm, Karl-Heinz Otto, Friedrich Schlette, Berthold Schmidt

Anschrift der Redaktion:

Zentralinstitut für Alte Geschichte und Archäologie der AdW,

Leipziger Str. 3-4, Postfach-Nr. 1310, DDR - 1086 Berlun, Fernruf : 2236266

Umschlagbild: Schwepnitz, Kr. Kamenz. Römische Silberdenare aus einem Schatzfund von 1985. (Foto: H. Boswank, Landesmuseum für Vorgeschichte Dresden)

Zeitschrift „Ausgrabungen und Funde“

Herausgeber: Zentralinstitut für Alte Geschichte und Archäologie

der Akademie der Wissenschaften der Deutschen Demokratischen Republik.

Verlag: A kademie-Verlag Berlin, DDR - Leipziger Straße 3-4, DDR - 1086 Berlin;

Fernruf: 2236229 und 2236201 . Telex-Nr.: 114420; Bank: Staatsbank der DDR, Berlin,

Kto.-Nr.: 6836-26-207 12.

Verantwortlicher Redảkteur: Prof. Dr. phil. habil. Joachim Herrmann.

Anschrift der Redaktion: Zentralinstitut für Alte Geschichte und Archäologie der AdW,

Leipziger Straße 3-4, Postfach 1310, DDR-1086 Berlin, Fernruf 2236266.

Veröffentlicht unter der Lizenznummer 1337 des Presseamtes beim Vorsitzenden des Ministerrates der Deutschen Demokratischen Republik. P 17/2,88 Z.

Gesamtherstellung: VEB Druckhaus „Maxim Gorki“, DDR - 7400 Altenburg.

Erscheinungsweise: Die Zeitschrift erscheint zweimonatlich. Die jährlich erscheinenden 6 Hefte bilden einen Band. Bezugspreis je Band 60,- DM zuzüglich Versandspesen. Preis je Heft 10,- DM. Der gültige Jahresbezugspreis für die DDR ist der Postzeitungsliste zu entnehmen.

Bestellnummer dieses Heftes 1038,33/1

(C) 1988 by Akademie-Verlag Berlin . Printed in the German Democratic Republic.

AN (EDV) 16920

00300 